\title{
Is Napping a Good Thing?
}

\author{
Sammar Atassi, MD ${ }^{1}$
}

\section{ABSTRACT}

This article aims to explore new findings in research in the relationship between sleep disorders and dementia. The new research questions the glymphatic system's role in clearing the toxic proteins from the brain during sleep.
Author affiliations are listed at the end of this article.

Correspondence to: Sammar Atassi, MD West Virginia University wvmedical@yahoo.com

\section{KEYWORDS}

Dementia, Mild Cognitive Impairment, Sleep Disorders, Glymphatic System

Until recently, excessive napping, which is common in dementia patients, was attributed to poor nighttime sleep caused by damage to the sleep centers in the brain. But new research indicates something otherwise. This sleep center, which includes alerting center regions in the brain, can be directly affected by dementia. Therefore, excessive napping may be a warning sign and precede memory loss before more significant symptoms of dementia develop.

Dementia afflicted patients are known to have sleep disturbances ranging from poor nighttime sleep, nighttime paranoia, and hallucinations, also known as sundown syndrome. Thus, daytime napping was generally considered a compensation to poor nighttime sleep caused directly by Alzheimer's disease.

There are two toxic amyloid proteins associated with dementia called beta-amyloid and tau. Both these proteins are metabolic waste products naturally present in the fluid between brain cells. In Alzheimer's disease, as NIH reports,' beta-amyloid clumps together to form amyloid plaques. These amyloid plaques then impair communication between neurons. Sleep disturbances then occur when these toxins are deposited in the regions of the brain that generate deep sleep.

The lack of sleep by itself can cause dementia. Recent research by Maiken Nedergaard, a neurologist at the University of Rochester Medical Center in New York, identified and described this phenomenon. ${ }^{2}$ There is a drainage system in the brain called the glymphatic system. This system circulates nutrients to the brain and washes away the waste products such as the amyloid proteins. This glymphatic system is most active at night during sleep. A new study done on mice in February 2019 showed that the deeper the sleep, the more active this washing system. ${ }^{3}$ Therefore, sleeping less than the minimum acceptable seven hours or having a sleep disorder that disturbs sleep, such as insomnia or obstructive sleep apnea, can disrupt this washing process. Unfortunately, this disruption of sleep and washing leads to the accumulation of toxic amyloid proteins in memory regions of the brain. 
Additionally, a new study released a few weeks ago from the University of California San Francisco found that deposits of the toxic tau protein in the regions of the brain that promotes wakefulness are causing neurodegeneration of the three main regions responsible for wakefulness. ${ }^{4}$ This occurs all at once and deprives the brain of any chance to compensate. The brain changes found in post-mortem studies preceded the typical symptoms of memory loss, though the researchers are not sure of the time-lapse between sleep disturbance and memory loss. This led researchers to the hypothesis that excessive napping in dementia patients could be an independent process by itself and not related to the sleep disturbance caused by Alzheimer's disease.

Approximately $10-15 \%$ of individuals with mild cognitive impairment with symptoms such as forgetting names, phone numbers, appointments, or losing the train of thought, are at a greater risk for developing dementia. ${ }^{5}$ People experiencing daytime sleepiness and frequent napping in the absence of a sleep disorder are at risk and should be screened for dementia.

\section{AUTHOR AFFILIATIONS}

1. West Virginia University, Morgantown, West Virginia

\section{REFERENCES}

1. National Institutes of Health. Lack of sleep may be linked to risk factor for Alzheimer's disease. US Department of Health \& Human Services. 2018.

2. Maiken Nedergaard. Available from: https://scholar.google.com/ citations? user=UtEXxxQAAAAJ\&hl=en

3. Hablitz LM et al. Increased glymphatic influx is correlated with high EEG delta power and low heart rate in mice under anesthesia. Science Advances. 2019;5(2):eaav5447.

4. Oh J et al. Profound degeneration of wakepromoting neurons in Alzheimer's disease. Alzheimer's \& Dementia. 2019;15(10):1253-1263.

5. Mayo Clinic. Mild cognitive impairment. Available from: https://www.mayoclinic.org/ diseases-conditions/mild-cognitive-impairment/ symptoms-causes/syc-20354578 